\title{
TÉCNICA Y EFECTIVIDAD BIOMECÁNICA DEL REMATE EN EL VOLEIBOL. REVISIÓN BIBLIOGRÁFICA
}

\section{TECHNICAL AND BIOMECHANICAL EFFECTIVITY TO SPIKE IN VOLLEYBALL. LITERATURE REVIEW}

\section{Quintana Salas, Jorge Iván}

Licenciado en Ciencias del Deporte y Actividad Física, Universidad Santo Tomás, Santiago, Chile.

Estudiante de Magister en Ciencias de la Actividad Física y el Deporte, Mención Rehabilitación y Entrenamiento Deportivo, Universidad Santo Tomás, Santiago, Chile.

QUINTANA S.J.I. (2015). La técnica y efectividad biomecánica del remate en el voleibol. Revisión bibliográfica. Mot Hum. 16 (1): 7-12.

\section{RESUMEN}

En el voleibol el remate es la acción de ataque más agresiva dentro del juego por medio del cual se obtienen la mayor cantidad de puntos. En la técnica de remate, es importante golpear el balón a la mayor altura posible e imprimirle velocidad y trayectoria. La efectividad desde el punto de vista biomecánico está dada por adquirir la mayor altura posible del centro de masa para golpear el balón lo más alto posible e imprimir velocidad y precisión en la trayectoria del balón. El objetivo del presente trabajo es ofrecer una revisión del conocimiento científico disponible en los últimos 20 años sobre el remate, con el fin de establecer el estado actual de los factores biomecánicos relacionados con su rendimiento e impulsar una visión crítica a los entrenadores generando herramientas científicas para planificar sus entrenamientos.

Los principales hallazgos de la presente revisión bibliográfica fueron, por un lado, la poca existencia de información sobre la técnica de remate del voleibol, y por otro lado, a pesar de existir un déficit de evidencia científica, las investigaciones se centran en los factores biomecánicos relacionados con los criterios de eficacia del remate, como la velocidad del centro de masa en la batida, la elevación del centro de masa, el tipo de armado, y la velocidad angular de la mano. En cuanto a los estudios de intervención analizados sobre el rendimiento del remate, el mayor número de publicaciones se obtuvo desde el 2011 a la actualidad, dejando en evidencia que el avance científico aun está en proceso.

Palabras claves: biomecánica, voleibol, remate.

\section{ABSTRACT}

In volleyball the spike is the most aggressive action attack in the game, in which the majority of points is obtained. In the art spike, it is important to hit the ball as high as possible and to give it speed and trajectory. Since the biomechanical point of view, the effect is given by acquiring the greatest possible height of the center of mass, and to hit the ball as high as possible to give speed and accuracy in the trajectory of the ball. The objective of this study is to provide a revision of the available scientific knowledge of the last 20 years about the spike with the only purpose of establishing the state of actual biomechanical factors in relation to its performance and promote a critical point of view to the coaches giving scientific tools to plan trainings.

The main findings of this literature review were, on the one hand, the poor information about the spike techniques in volleyball. On the other hand, despite on the luck of scientific evidences, the researcher focuses on biomechanical factors related to the performance criteria of the spike like the speed of the center of the mass in the impulse, the elevation of de center of the mass, type reinforced, and the angular velocity of the hand. About the studies of intervention analyzed on the performance of the spike, the highest number of publications was obtained since 2011 to nowadays, making it clear that scientist advancements are still in process.

Keywords: biomechanical, volleyball, spike. 


\section{INTRODUCCIÓN}

En el voleibol, se puede dividir el objetivo general del juego en dos ideas fundamentales, por una lado mantener el saque, por otro, recuperarlo. Aunque en la actualidad ambas suponen puntuar, los condicionantes para lograrlo provocan cambios estructurales notables entre una fase y otra. Para favorecer la comprensión de la estructura del voleibol y la dinámica de entrenamiento se divide clásicamente el desarrollo del juego en dos grandes fases. En primer lugar el complejo KI, protagonizado por las acciones que persiguen la obtención del punto cuando el saque está en posesión del adversario, también denominado fase de cambio o recuperación del saque, y en segundo lugar el complejo 2 o KII, responsable de preservar el saque o, lo que es lo mismo conseguir el punto cuando el saque está en posesión propia (Ureña et al., 2002). Al analizar las características del voleibol, este consiste en una realización de movimientos rápidos, acíclicos con alta intensidad y cortos intervalos de tiempo que tienen una duración promedio de 10-20 segundos (Bompa, 2002).

La acción del remate es el cuarto elemento del juego que tiene lugar dentro de la secuencia del KI. Supone por tanto el primer contacto netamente ofensivo que realiza un equipo para lograr un punto y la recuperación del saque. La importancia del remate perfecto contribuye al éxito del equipo en general. Sin una buena técnica de remate no se consiguen ataques rápidos o múltiples, con lo que estamos facilitando el juego de defensa del equipo contrario (Clemente et al., 2005).

El remate es el elemento que culmina la fase ofensiva de una jugada, para conseguirlo tiene que superar la altura de la red y la defensa contraria (Hernández, 1992).

Es la acción de juego con la que más puntos $\mathrm{s}$ e consiguen y la que más se correlaciona con el rendimiento (Palao et al., 2004). Algunos autores (Valadés et al., 2004), coinciden que el gesto técnico del remate está compuesto por una secuencia temporal de 5 fases: carrera de aproximación, batida, preparación para el golpeo, golpeo y por último la caída. Estas cinco etapas dan construcción a la acción más importante de juego en cuanto a la aportación de puntos, las cifras (Zhang, 2000) indican que el remate aporta más del $60 \%$ de los puntos convertidos por un equipo durante un partido.

La Biomecánica es una ciencia fundamental utilizada para ayudar a incrementar el rendimiento deportivo, y se rige por la aplicación de los principios técnicos de toda investigación en el análisis de la estructura, funciones y capacidades de los seres vivos en movimiento (Hernández, 2008). Por lo tanto, el propósito esencial de los estudios biomecánicos aplicados a cualquier destreza deportiva, en este caso correspondiente al remate de voleibol, se basa en demostrar en qué punto exacto el atleta ejecuta de forma errada un movimiento, afectando así su efectividad y el rendimiento de toda una selección. Valadés et al., (2007) indican que a nivel biomecánico, el rendimiento de la acción de remate se obtiene a partir de la optimización de tres factores: a) la altura de golpeo, b) la trayectoria del balón y c) la velocidad del balón tras el golpeo. Primero, la altura de golpeo depende de tres aspectos: a) la altura del Centro de Masa (CM) en el momento del despegue, b) la distancia de la mano al centro de gravedad en el momento del golpeo, y c) la altura del salto. Segundo, la trayectoria del balón en el remate depende de los movimientos del tronco, del hombro, de la cadera, del codo y de la muñeca, y del lugar de contacto del balón. Por último, la velocidad del balón en el remate depende directamente de la velocidad final de la mano de golpeo.

Por lo tanto, el objetivo del presente artículo consiste en ofrecer una revisión bibliográfica del conocimiento científico disponible en los últimos 20 años sobre el remate de voleibol, con el fin de establecer el estado actual de los factores biomecánicos relacionados con su rendimiento y así con los resultados obtenidos impulsar una visión crítica a los entrenadores, generando herramientas científicas para planificar mejor sus entrenamientos.

\section{MATERIAL Y MÉTODOS}

Las bases de datos utilizadas para realizar la búsqueda bibliográfica han sido PROQUEST, DIALNET, LATINDEX, SCIELO, BRASILEIRA DE BIOMECÁNICA y EBSCO. 
Las palabras claves utilizadas para la revisión bibliográfica han sido: «remate/spike», «Biomecánica/Biomechanical», todas ellas unidas al término «voleibol/volleyball».

Para focalizar al máximo la búsqueda se aplicaron varios filtros. En primer lugar, un límite temporal para limitar la búsqueda entre enero de 1995 y julio de 2015. Para la selección de artículos útiles para el estudio, éstos debían cumplir con los siguientes criterios de inclusión:

- Debían contener datos técnicos del voleibol.

- Debían investigar conceptos biomecánicos asociados al remate del voleibol (p. ej., velocidad, ángulos, etc.).

Tabla I: Revistas y bases de datos sobre diferentes estudios al utilizar los términos de búsqueda: «remate/spike», «Biomecánica/Biomechanical», todas ellas unidas al término «voleibol/volleyball», entre enero de 1995 y julio de 2015.
- Debían estar publicados en inglés, portugués o español.

\section{RESULTADOS}

Tras la búsqueda, se descartaron todos los artículos que no cumplían con los criterios de inclusión propuestos con anterioridad. De este modo, en la tabla 1 se puede visualizar a nivel cuantitativo el material encontrado en revistas y bases de datos donde queda en evidencia la poca generación de recursos científicos haciendo alusión a la técnica de remate en el voleibol.

\begin{tabular}{lc}
$\begin{array}{c}\text { Revistas / Bases De } \\
\text { Datos }\end{array}$ & Análisis De Estudios \\
\hline Proquest & 0 \\
Dialnet & 2 \\
Latindex & 0 \\
Scielo & 1 \\
Revista Brasileira de & \\
Biomecánica & 0 \\
Ebsco & 6 \\
\hline
\end{tabular}

Tabla II: Número de artículos publicados por años en el periodo transcurrido entre 1995 y la actualidad.

\begin{tabular}{lc}
$\begin{array}{c}\text { Artículos Publicados } \\
\text { Por Años }\end{array}$ & $\begin{array}{c}\text { Número De Estudios } \\
\text { Publicados }\end{array}$ \\
\hline $1995-2005$ & 2 \\
$2006-2010$ & 3 \\
$2011-$ Actualidad & 4 \\
\hline
\end{tabular}


Figura 1: Gráfico de referencias bibliográficas en años para el remate de voleibol desde una perspectiva biomecánica.

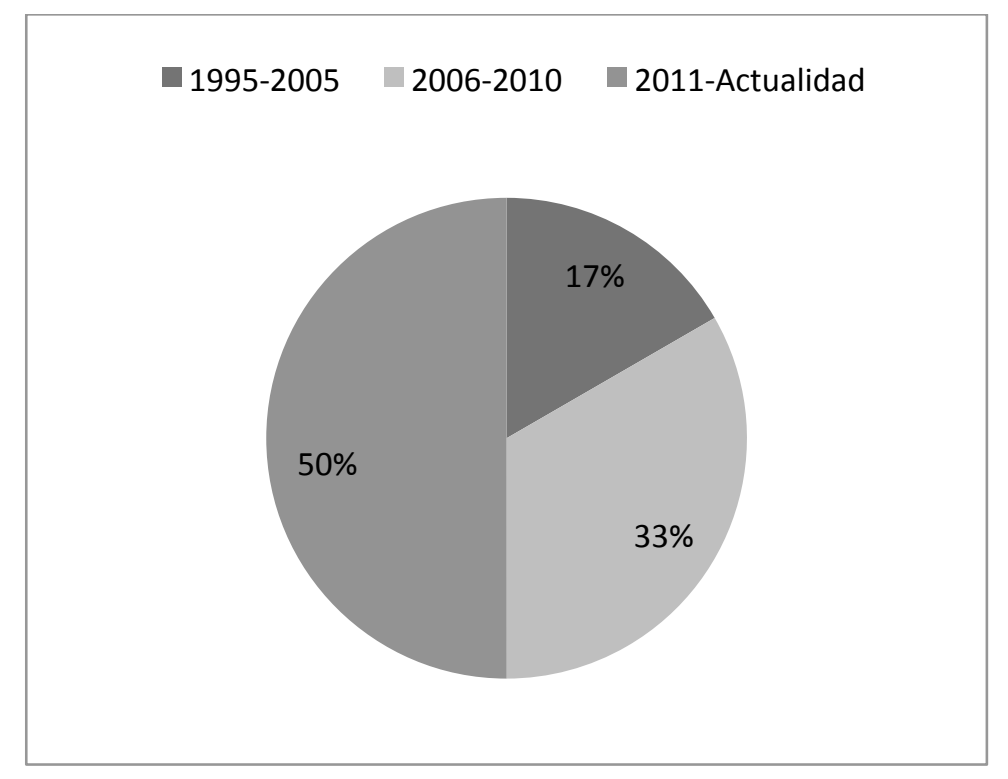

En la literatura actual se puede evidenciar que existe poco material bibliográfico sobre la técnica de remate con un enfoque biomecánica, sin embargo, aun faltan muchos estudios que complementen la información que se maneja en la actualidad. La investigación científica se ha guiado en mayor medida por el campo de la rehabilitación (lesiones musculo-esqueléticas), entrenamiento del salto (potencia y polimetría), entre otros. En la figura 1 podemos ver que el periodo donde se encuentra un mayor número de publicaciones se centra desde el 2011 a la actualidad, con un promedio de 3 publicaciones. Si bien, la cantidad de publicaciones es reducida, esto es un indicador fiable de que el avance científico sobre los gestos deportivos ha sido incluido como objetivo de estudio en el área de la biomecánica durante los últimos años.

\section{DISCUSIÓN}

A pesar de apreciar una gran variabilidad de información asociada al voleibol podemos determinar que las publicaciones de revistas se han relacionado a mayor frecuencia con temas relacionados a la rehabilitación (lesiones muscoesqueléticas), el entrenamiento del salto (potencia y pliometría), entre otros. Debido al déficit de información científica sobre la acción de remate desde una perspectiva biomecánica, tomaremos como referencia el estudio publicado por Bermejo et al., (2013) en el que se analizan valores de referencia cinemáticos del remate de voleibol en jugadoras de elite. En este estudio se argumenta que los factores biomecánicos relacionados con los criterios de eficacia del remate serian la velocidad del centro de masa en la batida (horizontal y vertical), la elevación del centro de masa durante el salto, el tipo de armado, y la velocidad angular de la mano. De esta manera, podemos afirmar que la biomecánica deportiva es una ciencia auxiliar del entrenamiento que se encarga de mejorar la técnica deportiva, con el fin de optimizarla y evitar las lesiones por sobrecarga corrigiendo su origen (García-Fojeda, 1997)

Autores como Vint, (1995); Ferragut et al., (2003); Valadés et al., (2005); Márquez et al., (2009), Márquez et al., (2011) centran sus estudios en el análisis de las fases impulso, armado y remate, dejando en evidencia la importancia de la biomecánica deportiva en el rendimiento eficaz del remate. Es así como en la fase de batida, Ferragut et al., (2003) indican que independiente a la realización o no de un contra-movimiento previo al salto, la altura de vuelo alcanzada en un salto vertical se puede predecir con gran exactitud a partir del impulso mecánico positivo, que es generado durante la fase de impulsión vertical hacia arriba. 
Según Márquez et al., (2009) en su estudio obtuvieron los siguientes valores para el centro de gravedad; la altura máxima 1,81 (m), la velocidad horizontal en el instante del primer contacto con el suelo $1,52(\mathrm{~m} / \mathrm{s})$ y la velocidad vertical en el instante del primer contacto con el suelo 3,69 (m/s). Siguiendo con la cadena cinética en la fase de preparación para el golpeo, Márquez et al., (2011) demuestran que los jugadores de voleibol giran de forma rápida e inclinan el tronco hacia delante $\mathrm{y}$ hacia la izquierda antes del impacto a la pelota. Estos movimientos hacia adelante crean momentos angulares hacia la izquierda del centro de gravedad. Sin embargo, dado que el cuerpo está en el aire, y que se sigue la ley de acción-reacción y la ley de la conservación del momento angular, la parte inferior del cuerpo lo hará reaccionar simultáneamente flexionando ambas caderas. Según Vint (1995) la velocidad que la mano le transmitía al balón, dependía en un $46 \%$ de la extensión del codo, en un $20,5 \%$ de la rotación del hombro, en un $14,5 \%$ de la actuación de rotación del tronco, en un 7,5\% del desplazamiento hacia adelante del centro de gravedad durante el salto, en un 5,5\% de la flexión de la muñeca y el $6 \%$ restante dependía de otros factores. Finalmente, para la fase de golpeo, Valadés et al., (2005) argumentan que durante la acción, es preciso evitar la pérdida de energía generada en la mano, la energía perdida en el contacto depende de las características de deformación tanto en la mano como del balón.

Cuanto más rígida esté la mano y más duro el balón, menos energía se perderá en el golpeo. Es por esta razón que las manos están en forma de copa con los dedos separados para no reducir su rigidez. Además Mitchinson et al., (2013) en su estudio indican que la velocidad del balón obtenida en sujetos ilesos es de $19,0 \mathrm{~m} / \mathrm{s}$. y en sujetos lesionados de 19,4 m/s.

Por último, es importante indicar que a pesar de que se han revisado de forma exhaustiva todos los artículos publicados sobre la biomecánica del remate de voleibol, creemos en el presente trabajo de investigación tiene algunas limitaciones. Los estudios elegidos para esta revisión proceden de bases de datos electrónicas o publicaciones de acceso gratuito, por ende todos los artículos de otras revistas de alto impacto (Pubmed, International Society of Biomechanics, etc.) en cuales se cancela por sus servicios, se han excluido. Además, tampoco se han incluido los artículos publicados en otros idiomas que no sean inglés, portugués o español.

\section{REFERENCIAS BIBLIOGRÁFICAS}

Bermejo J, Pelao J, Valades D. (2013). Analysis of volleyball spike in female elite players. Agon Internacional journal of Sport Sciences, 3(1), 22-32.

Bompa, T. (2002). Periodização: teoria e metodologia do treinamento. São Paulo: Ed. Phorte. Clemente J, Antonio J, Moreno Domínguez A, Moreno Arroyo P. (2005). Estudio de la relación entre la eficacia de las acciones de primer contacto y la eficacia del ataque en voleibol masculino de alto nivel. Kronos: revista universitaria de la actividad física y el deporte, (8): $57-61$

Ferragut C, Cortadellas J, Arteaga R, Calbet JAL. (2003). Predicción de la altura de salto vertical. Importancia del impulso mecánico y de la masa muscular de las extremidades inferiores. European Journal of Human Movement, 10: 7-22.

García-Fojeda A, Biosca F, Vàlios J. (1997). La biomecánica: una herramienta para la evaluación de la técnica deportiva. Educación Física y Deportes, (47): 15-20.

Hernández A. (2008). Mecánica Newtoniana y deporte, movimiento de los proyectiles. Consejo de publicaciones. Universidad de Los Andes. Mérida Venezuela.

Hernández, L. (1992). La técnica. En COE (Ed). Voleibol. C.O.E. Madrid (pp.59-132).

Marquez W, Masumura M, Ae M. (2009). The effects of jumping distance on the landing mechanics after a volleyball spike. Sports Biomechanics; 8 (2): 154-66.

Marquez W, Masumura M, Ae M. (2011). Spike landing motion of elite male volleyball players during official games. International Journal of Sport \& Health Science, (9): 82-89.

Mitchinson L, Campbell A, Oldmeadow D, Gibson W, Hopper D. (2013).

Comparison of upper arm kinematics during a volleyball spike between players with and without a historyof shoulder injury. Journal of Applied Biomechanics, (29): 155-164.

Palao J, Santos J, Ureña A. (2004). Effect of team level on skill performance in volleyball. International Journal of Performance Analysis in Sport, 4(2): 50-60. 
Ureña A, Calvo R, Lozano C. (2002). Estudio de la recepción del saque en el voleibol masculino español de elite tras la incorporación del jugador libero. Revista Internacional de Medicina y Ciencias de la Actividad Física y del Deporte, (4), 3.

Valadés D, Palao J, Femia P, Radial P, Ureña A. (2007). Validez y fiabilidad del radar para el control de la velocidad del remate en voleibol. Cultura, Ciencia y Deporte, 6 (2): 131138.

Valades D. (2005). Efecto de un entrenamiento en el tren superior basado en el ciclo estiramiento - acortamiento sobre la velocidad del balón en el remate de Voleibol. Editorial de la Universidad de Granada. España.

Vint, P. (1994). The mechanics of motion: Scientific aspects of jumping. Coaching Volleyball, 26-27.
Vint, P. (1995). Secrets of speed: An in depth look at spiking. Performance Conditioning for Volleyball, 2(9), 4-6.

Zhang R. (2000). How to profit by the new rules. The Co ach, 1: 9-11.

\section{Dirigir Correspondencia a:}

Jorge Iván Quintana Salas

Pasaje Bernini \# 2376, La Florida, Región

Metropolitana de Santiago, CHILE.

Fono: (2) 22712289

Email-jorgequintanasalas@gmail.com

RECIBIDO: 8 de Junio de 2015

ACEPTADO: 15 de Junio de 2015 